\title{
Characterizing Cortex-Wide Dynamics with Wide-Field Calcium Imaging
}

\author{
Chi Ren and ${ }^{\mathbb{D}}$ Takaki Komiyama \\ Neurobiology Section, Center for Neural Circuits and Behavior, Department of Neurosciences, and Halıcıŏlu Data Science Institute, University of \\ California San Diego, La Jolla, California 92093
}

The brain functions through coordinated activity among distributed regions. Wide-field calcium imaging, combined with improved genetically encoded calcium indicators, allows sufficient signal-to-noise ratio and spatiotemporal resolution to afford a unique opportunity to capture cortex-wide dynamics on a moment-by-moment basis in behaving animals. Recent applications of this approach have been uncovering cortical dynamics at unprecedented scales during various cognitive processes, ranging from relatively simple sensorimotor integration to more complex decision-making tasks. In this review, we will highlight recent scientific advances enabled by wide-field calcium imaging in behaving mice. We then summarize several technical considerations and future opportunities for wide-field imaging to uncover large-scale circuit dynamics.

Key words: decision making; large-scale cortical dynamics; learning; multimodal recordings; sensorimotor integration; wide-field calcium imaging

\section{Introduction}

The brain is a modular structure in which communication across multiple regions functions to drive behavior and cognition. The emergent properties of such macroscopic interactions cannot be deduced simply by characterizing individual brain regions separately. Therefore, to better understand how the brain functions as a whole, it is critical to record from multiple brain regions simultaneously. Wide-field functional imaging is well suited for this purpose. In systems neuroscience, wide-field calcium imaging has been used to record activity across broad brain areas simultaneously through one-photon excitation (Cardin et al., 2020). Although this technique normally does not resolve single cells, it enables simultaneous capturing of neural dynamics across brain areas with a sufficient spatial and temporal resolution to resolve behaviorally relevant information (for comparisons of various large-scale imaging modalities, see Table 1). This review will mainly focus on macroscale wide-field calcium imaging applied to most of the dorsal cortex in mice. Similar approaches are also called "mesoscale" and "mesoscopic," often emphasizing the spatial resolution that can resolve subregions within individual brain areas but does not achieve single-cell resolution.

Wide-field functional imaging has traditionally been achieved by measuring the "intrinsic signal" or using fluorescent voltagesensitive dyes. Intrinsic signals are changes in optical reflectance caused by changes in blood volume and oxygenation which correlate with local neural activity (Berwick et al., 2005; Ma et al., 2016b; Mateo et al., 2017). Unlike intrinsic signals, voltage-

\footnotetext{
Received Dec. 1, 2020; revised Mar. 26, 2021; accepted Mar. 30, 2021.

This work was supported by National Institutes of Health Grants R01 NS091010, R01 EY025349, R01 DC014690, and P30 EY022589, National Science Foundation 1940202, and David \& Lucile Packard Foundation to T.K.

The authors declare no competing financial interests.

Correspondence should be addressed to Takaki Komiyama at tkomiyama@ucsd.edu.

https://doi.org/10.1523/JNEUROSCl.3003-20.2021

Copyright $\odot 2021$ the authors
}

sensitive dyes serve as direct indicators of neural activity by responding to membrane potential changes; furthermore, they provide a higher temporal resolution owing to their faster kinetics (Orbach et al., 1985; Grinvald and Hildesheim, 2004). Although both approaches have been used to characterize largescale functional properties of cortex (Blasdel and Salama, 1986; Grinvald et al., 1986; Frostig et al., 1990; Bonhoeffer and Grinvald, 1991; Prechtl et al., 1997; Mohajerani et al., 2010), their ability to capture cortical dynamics is limited because of relatively low signal-to-noise ratio (SNR). Therefore, extracting activity patterns often relies on averaging over repeated measurements, ignoring the variability in momentby-moment interactions between cortical regions.

In recent years, the application of wide-field imaging in systems neuroscience has been revolutionized with the improvement of genetically encoded fluorescent indicators. These engineered proteins change the fluorescence intensity in response to a variety of neuronal events, including transmembrane voltage, intracellular calcium concentration, vesicle release, and changes in neurotransmitter concentration (Lin and Schnitzer, 2016; Sabatini and Tian, 2020). Among these protein sensors, genetically encoded calcium indicators, especially the GCaMP family (Tian et al., 2009; Akerboom et al., 2012; T. W. Chen et al., 2013; X. R. Sun et al., 2013; Y. Yang et al., 2018; Dana et al., 2019), have become a standard choice to visualize neural activity in both one-photon and multiphoton imaging. GCaMP fluorescence is sensitive to changes in intracellular calcium dynamics that are dominated by action potentials and thus reports neuronal spiking activity with high SNR. Genetic encoding of GCaMP also enables stable expression over time for longitudinal recordings. These advantages of GCaMP allow wide-field calcium imaging to overcome the difficulties often encountered with intrinsic signal imaging and voltage-sensitive dye imaging, making it an attractive approach to characterize large-scale cortical dynamics in behaving animals. 
Table 1. Comparison of several large-scale imaging modalities in mice based on the parameters typically used in recent studies ${ }^{a}$

\begin{tabular}{|c|c|c|c|c|c|}
\hline & Wide-field calcium imaging & Other wide-field functional imaging & Large-scale two-photon calcium imaging & 3D volumetric two-photon calcium imaging & BOLD fMRI \\
\hline FOV & $\begin{array}{l}\sim 10 \times 10 \mathrm{~mm}^{2} \text { to cover most of the dorsal } \\
\quad \text { cortex }\end{array}$ & $\begin{array}{l}\sim 10 \times 10 \mathrm{~mm}^{2} \text { to cover most of the dorsal } \\
\quad \text { cortex }\end{array}$ & $\sim 5 \times 5 \mathrm{~mm}^{2}$ & $\begin{array}{l}\text { Varying across different designs and studies, } \\
\text { typically } \sim 0.16-1 \mathrm{~mm} 2 \times 100- \\
600 \mu \mathrm{m} \text { axial range }\end{array}$ & Whole brain \\
\hline Spatial resolution & $\sim 10-100 \mu \mathrm{m} /$ pixel & $\sim 10-100 \mu \mathrm{m} /$ pixel & Cellular or subcellular resolution & Cellular or subcellular resolution & $\begin{array}{r}\sim 0.2-0.4 \times 0.2-0.4 \times 0.5-1.2 \\
\mathrm{~mm}^{3} / \text { voxel in recent studies }\end{array}$ \\
\hline $\begin{array}{l}\text { Temporal resolution/sampling } \\
\text { frequency }\end{array}$ & $\begin{array}{l}\sim 30 \mathrm{~Hz} \text {, actual temporal resolution may be } \\
\text { lower ( } \sim 50-100 \mathrm{~ms}) \text { because of slow } \\
\text { kinetics of some indicators }\end{array}$ & $\begin{array}{l}\sim 30 \mathrm{~Hz} \text { for intrinsic signal imaging, actual } \\
\text { temporal resolution may be lower } \\
(\sim 100 \mathrm{~ms}) \text { because of slow kinetics of } \\
\text { intrinsic signals; } \sim 0.1-1 \mathrm{kHz} \text { for volt- } \\
\text { age-sensitive dye imaging }\end{array}$ & $\begin{array}{l}\text { Varying across different designs and studies, } \\
\text { ranging from } 0.1-7.5 \mathrm{~Hz} \text { to scan the } \\
\text { whole FOV with cellular resolution }\end{array}$ & $\begin{array}{l}\text { Varying across different designs and studies, } \\
\text { usually } \sim 10-50 \mathrm{~Hz}\end{array}$ & $\sim 1 \mathrm{~s}$ \\
\hline Recording depth & Superficial layers, $<\sim 200 \mu \mathrm{m}$ & Superficial layers, $<\sim 200 \mu \mathrm{m}$ & Up to $\sim 600 \mu \mathrm{m}$ & Up to $\sim 600 \mu \mathrm{m}$ & Whole brain \\
\hline Optics/camera/lens requirement & $\begin{array}{l}\text { Custom-built or commercial fluorescence } \\
\text { stereo microscopes and CCD or CMOS } \\
\text { cameras }\end{array}$ & $\begin{array}{l}\text { Custom-built or commercial fluorescence } \\
\text { stereo microscopes and CCD or CMOS } \\
\text { cameras }\end{array}$ & $\begin{array}{l}\text { Custom-built or commercial two-photon } \\
\text { microscopes with wide FOV and ran- } \\
\text { dom-access scanning }\end{array}$ & $\begin{array}{l}\text { Custom-built or commercial two-photon } \\
\text { microscopes with multidepth scanning, } \\
\text { using, e.g., deformable mirror, spatial } \\
\text { light modulator, Bessel optical module, } \\
\text { and variable-focus lens }\end{array}$ & Commercial systems \\
\hline Selected references & $\begin{array}{l}\text { Makino et al., 2017; Musall et al., 2019; } \\
\text { Pinto et al., 2019; Peters et al., } 2021\end{array}$ & $\begin{array}{l}\text { Bauer et al., 2014; Kyriakatos et al., 2017; } \\
\text { Kura et al., 2018; Karimi Abadchi et al., } \\
2020\end{array}$ & $\begin{array}{l}\text { Sofroniew et al., 2016; Stirman et al., 2016; } \\
\text { 0ta, 2020; Yu et al., } 2020\end{array}$ & $\begin{array}{l}\text { Ji et al., 2016; Nöbauer et al., 2017; Song } \\
\text { et al., 2017; W. Yang et al., 2018; } \\
\text { Weisenburger et al., 2019; Lu et al., } \\
2020\end{array}$ & $\begin{array}{l}\text { Schwalm et al., 2017; Schlegel et al., } \\
\text { 2018; Jung et al., 2019; Lake et } \\
\text { al., } 2020\end{array}$ \\
\hline
\end{tabular}

${ }^{a}$ Within each modality, higher sampling frequency can be achieved at the sacrifice of spatial resolution and vice versa.

Several studies have conducted one-photon calcium imaging with GCaMP at a mesoscale level with the field of view (FOV) covering several adjacent cortical regions in adult animals (Vanni and Murphy, 2014; Niethard et al., 2016; Wekselblatt et al., 2016; T. W. Chen et al., 2017; Zhuang et al., 2017). This approach has also been used to investigate the developing circuits in both cortex and subcortical regions (Ackman et al., 2012; Burbridge et al., 2014; Gribizis et al., 2019). Meanwhile, a growing list of studies use wide-field calcium imaging to characterize cortical activity at a macroscopic level with an FOV encompassing most of the mouse dorsal cortex (Fig. 1). Such studies have deepened our understanding of cortex-wide dynamics in various cognitive processes, ranging from relatively simple sensorimotor integration to more complex decision-making tasks (Allen et al., 2017; Makino et al., 2017; Gilad et al., 2018; Musall et al., 2019; Pinto et al., 2019; Shimaoka et al., 2019; Gilad and Helmchen, 2020; Salkoff et al., 2020). In this review, we first focus on recent studies performing widefield calcium imaging in behaving mice. Using these example studies, we highlight the versatility of wide-field calcium imaging for revealing novel insights into various questions. We then discuss several technical considerations for widefield calcium imaging. Finally, we discuss future opportunities for the development and application of wide-field imaging to uncover large-scale circuit dynamics.

\section{Propagation of cortical activity in sensorimotor integration}

Generating appropriate actions requires integrating sensory information from the environment, and such sensorimotor processing often recruits distributed brain regions to achieve precise sensory perception, action selection, and movement execution. The spatiotemporal dynamics of large-scale cortical activity during sensorimotor transformation have been studied extensively in the rodent whisker system (Ferezou et al., 2007; Matyas et al., 2010; Kyriakatos et al., 2017; Sreenivasan et al., 2017; Gilad et al., 2018). A series of studies using wide-field voltage-sensitive dye imaging has revealed that a single whisker deflection evokes a highly distributed cortical sensory response, starting in barrel cortex and then propagating to primary motor cortex, to drive whisker movements (Ferezou et al., 2007; Matyas et al., 2010; Kyriakatos et al., 2017). The spread of the sensory response is attenuated during active whisking, when the animal's ability to detect weak stimuli is impaired, suggesting that the distributed sensory response is dynamically modulated by ongoing behavior (Ferezou et al., 2007; Kyriakatos et al., 2017). With wide-field calcium imaging, Gilad et al. (2018) further investigated the macroscopic cortical dynamics under different behavior strategies in a whisker-based texture discrimination task with delayed actions to report the choice (lick or no lick). During the delay period between the texture sensation and the chosen action, the activation of different cortical regions, especially the secondary motor cortex (M2) and a posterior cortical region area $\mathrm{P}$, was contingent on the behavioral strategies animals deployed to solve the task. When mice took an active strategy (engaging their body toward the approaching texture), M2 showed sustained activity during the delay period, holding information about the future action. In contrast, in mice using a passive strategy in which they quietly awaited the texture touch, area $\mathrm{P}$ displayed enhanced activity during the delay period, holding information about the stimulus identity. Furthermore, optogenetic inactivation of M2 and area $\mathrm{P}$ during the delay period led to impairment in behavioral performance during active and passive strategies, respectively. These results support the model that cortical activity can be dynamically routed to different regions to hold the task-relevant information before converging to similar chosen actions (Gilad et al., 2018). It is worth noting that the unbiased observation with wide-field calcium imaging revealed a novel role of area $\mathrm{P}$ in texture discrimination. Area $\mathrm{P}$ has been mainly implicated in visual processing in previous literature (Garrett et al., 2014; Zhuang et al., 2017), and its function in tactile texture discrimination suggests that it may be generally involved in processing information related to object identity (Gilad et al., 2018). With wide-field imaging, these studies provide the first glimpse of the macroscopic activity pattern during sensorimotor 

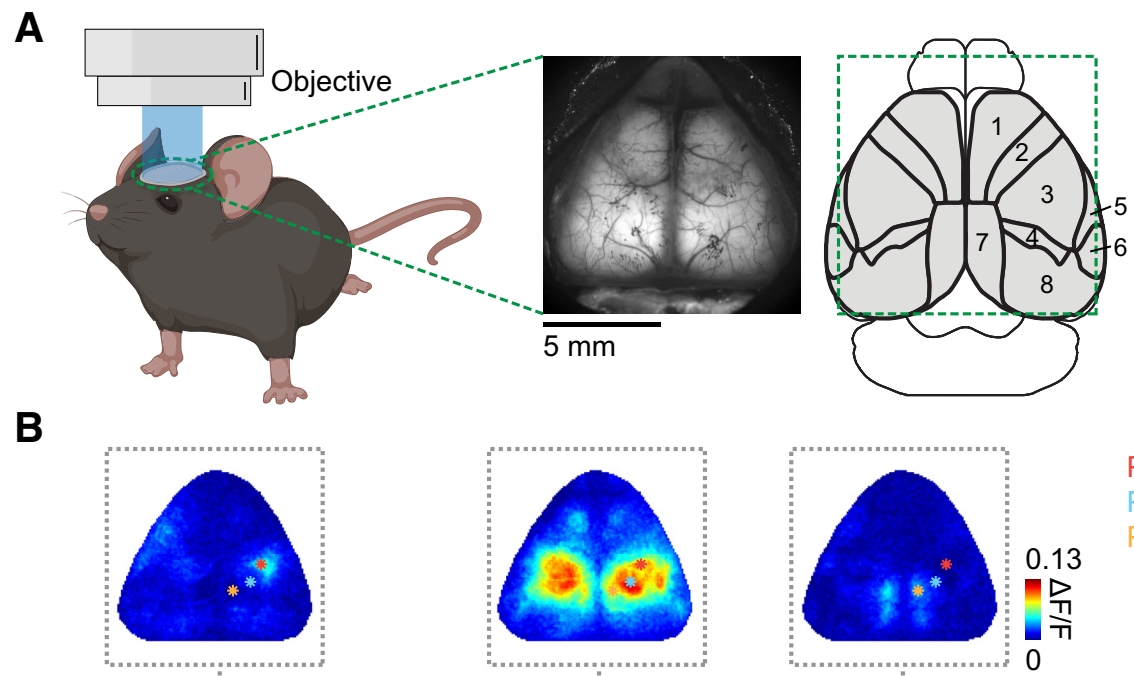

Pixel 1

Pixel 2 Pixel 3

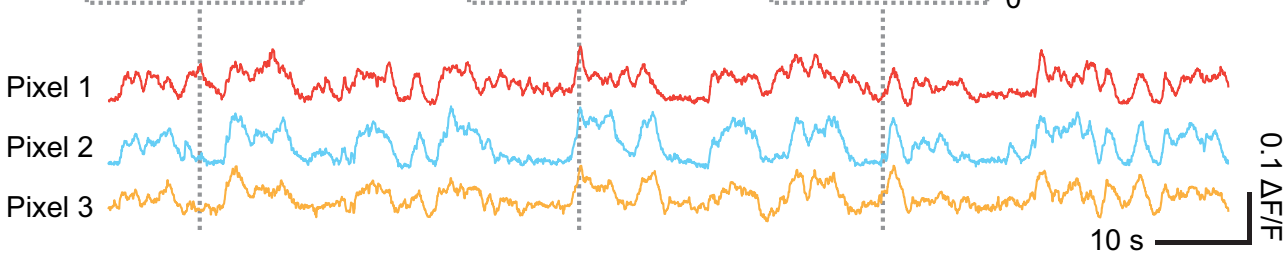

Figure 1. Wide-field calcium imaging of cortex-wide activity. A, Left, Imaging setup. Middle, An FOV of wide-field calcium imaging in a mouse cortex expressing GCaMP6s in cortical excitatory neurons. Right, Cortical regions (based on the mouse brain atlas from the Allen Institute) simultaneously recorded by wide-field calcium imaging. Green dashed box represents the area within the FOV. M2, Secondary motor cortex; M1, primary motor cortex; S1, primary somatosensory cortex; PPC, posterior parietal cortex; S2, secondary somatosensory cortex; Aud., auditory cortex; RSC, retrosplenial cortex; Vis., visual cortex. B, Example cortex-wide image frames and fluorescence traces of individual pixels in a behaving mouse. Gray dashed lines indicate the time of image frames in fluorescence traces of example pixels.

integration and demonstrate its fundamental flexibility even in simple sensorimotor processing.

\section{Distributed encoding of different types of information in cortex}

The distributed activation of many brain areas has been observed in various sensorimotor tasks (Goard et al., 2016; Allen et al., 2017; Kyriakatos et al., 2017; Makino et al., 2017; Gilad et al., 2018; Hattori et al., 2019; Musall et al., 2019; Pinto et al., 2019; Shimaoka et al., 2019; Steinmetz et al., 2019; Gilad and Helmchen, 2020; Salkoff et al., 2020); however, systematic optogenetic inactivation generally localizes behavioral effects to only a few regions (Guo et al., 2014; Goard et al., 2016; Allen et al., 2017; Pinto et al., 2019; Zatka-Haas et al., 2020). Therefore, it is important to resolve the information represented in each cortical region and its relevance to the ongoing behavior. Compared with wide-field imaging using intrinsic signals or voltage-sensitive dyes, the higher SNR of wide-field calcium imaging enables a detailed examination of information encoded in cortical activity using regression and decoding analyses on a trial-by-trial or moment-by-moment basis, without averaging out the behaviorally relevant variability (Allen et al., 2017; Gilad et al., 2018; Musall et al., 2019; Pinto et al., 2019; Salkoff et al., 2020; ZatkaHaas et al., 2020). By monitoring a variety of behavioral information and task events encoded in cortex-wide activity, researchers are able to systematically relate behavioral processes to neural activity (Musall et al., 2019; Shimaoka et al., 2019; Salkoff et al., 2020; Zatka-Haas et al., 2020).

Task-relevant information, such as sensory stimuli and choice, is represented in distributed but specific sets of cortical regions, generating distinct cortical activity patterns during task performance (Gilad et al., 2018; Musall et al., 2019; Pinto et al.,
2019; Salkoff et al., 2020; Zatka-Haas et al., 2020). Furthermore, the cortical activity pattern is modulated by task demands. Tasks with complex cognitive demands evoked activity profiles that were more different across cortical regions and engaged more spatially distributed information processing in the cortex (Pinto et al., 2019). For example, the encoding of sensory and choice information was more distributed in evidence accumulation or memory-guided tasks than simple perceptual decision-making tasks (Pinto et al., 2019; Salkoff et al., 2020; Zatka-Haas et al., 2020). The widespread cortical involvement in more demanding tasks was further confirmed with optogenetic inactivation (Pinto et al., 2019). These results suggest that the representation of taskrelevant information in the large-scale cortical network is dynamically modulated by the cognitive processes required in different tasks, and more complex cognitive processes engage more spatially distributed computations across the cortex.

In contrast to task-relevant information, movement is represented in widespread areas of the dorsal cortex regardless of the task complexity (Musall et al., 2019; Shimaoka et al., 2019; Salkoff et al., 2020; Zatka-Haas et al., 2020) and learning stage (Musall et al., 2019). The widespread dominance of movementrelated information in cortex has also been observed in spontaneous activity recorded with two-photon calcium imaging and in electrophysiological data collected from multiple brain regions during task performance (Steinmetz et al., 2019; Stringer et al., 2019). The prevalent encoding of movement can precede movement onset, arising in the primary motor cortex and expanding to the rest of cortical regions before movement (Zatka-Haas et al., 2020), suggesting an origin from efference copy of the motor command rather than sensory feedback generated by the movement. Further investigation revealed that uninstructed movements, which were not required for the task but spontaneously 
made by the animals, better explained the trial-by-trial variability in cortex-wide activity than instructed movements and task events. Meanwhile, uninstructed movements could also become correlated with instructed movements and stereotypically occurred around task events, affecting the trial-averaged neural activity (Musall et al., 2019). Although the function of such prevalent encoding of movements, if any, needs further investigation, the profound impact of movements on neural activity has raised the importance of careful behavioral monitoring in the interpretation of neural activity, especially for choices associated with asymmetric motor outputs (e.g., Go/NoGo tasks).

\section{Learning-related dynamics in macroscopic cortical activity}

Learning-induced plasticity has been under intense scrutiny with electrophysiological recordings and two-photon calcium imaging (Costa et al., 2004; Peters et al., 2014; Makino and Komiyama, 2015; Grewe et al., 2017). Most of these investigations have focused on the plasticity of local circuits in only one or a few brain regions, omitting one important piece of the puzzle: the interaction across many brain regions during learning. Taking advantage of the stable expression of genetically encoded calcium indicators over time, several recent studies have performed longitudinal wide-field calcium imaging to investigate learningrelated macroscopic dynamics (Makino et al., 2017; Musall et al., 2019; Gilad and Helmchen, 2020). Makino et al. (2017) systematically characterized the reconfiguration of cortex-wide activity during motor learning. Consistent with what we have discussed in previous sections, motor learning evoked distributed activation of most of the cortex, forming a macroscopic sequential activity. With learning, this macroscopic sequence of activity during movement execution became more temporally compressed and reproducible from trial to trial, suggesting that more efficient and reliable signal transmission across cortical regions evolves as a function of learning. At the same time, learning rerouted the cortical activity flow. With learning, a novel activity stream originated from M2 and flowed to the rest of the cortex, and the activity of M2 became predictive of the activity of other cortical regions on a moment-by-moment basis. The novel function acquired by $\mathrm{M} 2$ during learning was further confirmed with perturbation experiments. Bilateral M2 inactivation with muscimol in expert animals reversed both the cortical dynamics and behavioral performance toward the naive stage of learning, suggesting an indispensable role of M2 in coordinating cortex-wide dynamics for learned behavior (Makino et al., 2017).

The reorganization of cortex-wide activity is not unique to motor learning. Gilad and Helmchen (2020) reported a spatiotemporal refinement of cortex-wide activity flow in an associative learning task, where mice learned to report different textures through licking. At the early stage of learning, task engagement induced a general suppression in association cortices in the interval between the auditory cue signaling the trial start and the whisker-texture touch (the "pre-period"). As learning proceeded, activation increased in rostro-lateral cortex (part of the posterior parietal cortex) and the barrel cortex during the pre-period, building up an anticipatory activity stream arising in rostro-lateral cortex and flowing to the barrel cortex immediately. The specific enhancement of task-related cortical activation emerged in parallel with improved task performance and could contribute to the improved discrimination between different textures (Gilad and Helmchen, 2020). The cortex-wide dynamics observed in different learning tasks demonstrate that the learning-induced plasticity is not only confined to individual cortical regions separately, but also involves cortex-wide changes in the interaction between regions. Such reconfiguration of the large-scale cortical network during learning often involves association cortices and eventually produces more efficient processing of relevant information and more stable representations of learned behaviors.

\section{Multimodal recordings with wide-field calcium imaging Combining wide-field calcium imaging with complementary imaging modalities}

Although wide-field calcium imaging has revealed many novel features of macroscopic cortical dynamics, its current applications are still restricted by two major factors: the lack of singlecell resolution and limited recording depth in brain tissue. These limitations can be mitigated by combining wide-field calcium imaging with other imaging modalities, such as two-photon calcium imaging and fMRI (Barson et al., 2020; Lake et al., 2020). Barson et al. (2020) successfully performed simultaneous widefield and two-photon calcium imaging in awake mice. To avoid interference between the two imaging modalities, the light path of two-photon calcium imaging was redirected through a microprism mounted on the cortical surface. This multimodal setup is particularly advantageous for investigating the relationships between individual neurons and the entire cortex. For example, Barson et al. (2020) found that the activity of individual neurons in the same cortical region coincided with diverse cortex-wide activity patterns, such that different neurons correlated with different cortex-wide activity patterns. The activity of neighboring neurons can couple with distinct cortical activity patterns, which may arise from different anatomic connectivity. Furthermore, the association between the activity of individual neurons and cortex-wide activity can be modulated by behavioral states (Barson et al., 2020). These results suggest diverse and dynamic associations between local and global neural networks, where information can be dynamically routed depending on behavioral contexts and cognitive processes.

To complement the limited accessibility in the recording depth of wide-field calcium imaging, Lake et al. (2020) combined wide-field calcium imaging and fMRI, which allows simultaneous recording of large-scale cortical and subcortical activity. They found that calcium signals from excitatory neurons partially explained the variance in fMRI BOLD signals. Since the fMRI BOLD signal is an indiscriminate representation of integrated brain activity while wide-field calcium imaging can achieve cell type specificity, this multimodal recording setup could be instrumental in quantifying the contributions of different cell types to the fMRI BOLD signal (Lake et al., 2020).

\section{Combining wide-field calcium imaging with electrophysiological recordings}

The relatively simple surgical preparation and imaging setup make wide-field calcium imaging a feasible platform to be combined with electrophysiological recordings. To minimize obstruction of the FOV in wide-field imaging, this combination can be achieved by either inserting a traditional probe (e.g., glass pipette or silicon probe) at an angle (Xiao et al., 2017; Clancy et al., 2019; Peters et al., 2021) or using a flexible transparent probe (Liu et al., 2021). This multimodal recoding setup has been effective in investigating the relationships between cortical or subcortical single-neuron activity and large-scale cortical activity (Xiao et al., 2017; Clancy et al., 2019), as well as the communication between the cortex and subcortical regions (Liu et al., 2021; Peters et al., 2021). Consistent with observations from simultaneous wide-field and two-photon calcium imaging (Barson et al., 2020), multimodal recordings combining wide-field calcium 
imaging with electrophysiological recordings revealed that the cortex-wide activity patterns associated with single cortical or subcortical neurons were variable from neuron to neuron and modulated by behavior states (Xiao et al., 2017; Clancy et al., 2019).

A more systematic characterization of the functional mapping between cortex and subcortical regions was recently achieved with the Neuropixel probe, which significantly boosted the sampling power of electrophysiological recordings. By simultaneously recording in the cortex with wide-field calcium imaging and in the striatum with Neuropixel probes, Peters et al. (2021) revealed a topographical mapping between cortical and striatal activity. This functional mapping was consistent with the anatomic corticostriatal projections and independent of the animal's behavior states, suggesting that corticostriatal projections reliably propagate cortical activity to the associated striatal regions regardless of the behavioral state (Peters et al., 2021).

In addition to functional mapping, pairing wide-field calcium imaging with electrophysiological recordings can capture realtime interactions between cortex and subcortical regions. Liu et al., 2021 characterized the coordination between the cortex and the hippocampus during awake hippocampal sharp-wave ripples using a newly developed flexible transparent probe (NeuroFITM). They found that diverse patterns of cortex-wide activity accompanied sharp-wave ripples. In contrast to the conventional view that cortical activity is mainly triggered by hippocampal sharp-wave ripples, the cortical activation preceded hippocampal sharp-wave ripples in a majority of cases. Furthermore, the ongoing cortical patterns could be decoded from the spiking activity of hippocampal neuron populations, indicating a predictable relationship between cortical and hippocampal activity patterns. These results support the model that the hippocampus and the cortex interact during sharp-wave ripples in a selective and diverse manner at the macroscale (Liu et al., 2021).

Combining wide-field calcium imaging and other recording modalities extends the application of wide-field calcium imaging in at least two aspects. First, it bridges the gap between neural activity at different spatial scales and helps study how local circuits relate to larger neural networks (Xiao et al., 2017; Clancy et al., 2019; Barson et al., 2020). As typical two-photon calcium imaging and electrophysiological recordings often focus on a single brain area, investigations of the relationship between individual neurons and the larger brain network will contribute to a more comprehensive interpretation of local neural dynamics. Second, it compensates for the limited accessibility in the recording depth of wide-field calcium imaging and offers an attractive platform to investigate the dynamics of large-scale neural networks spanning the cortex and subcortical regions during various cognitive processes (Lake et al., 2020; Liu et al., 2021; Peters et al., 2021).

\section{Technical considerations of wide-field calcium imaging}

Although wide-field calcium imaging is a powerful tool for monitoring large-scale cortical dynamics and the technique per se is relatively simple to set up using conventional wide-field microscopes, several considerations should be kept in mind in the implementation of wide-field calcium imaging. First, wide-field calcium signals are likely dominated by activity from superficial cortical layers because of the strong scattering of both excitation and emission light in brain tissue. In one-photon excitation, the intensity of excitation light $(\sim 480 \mathrm{~nm})$ of GCaMP already drops to $\sim 10 \%$ at a depth of $200 \mu \mathrm{m}$ (Yizhar et al., 2011), suggesting that most signals come from cortical layer 1 and layer $2 / 3$. Second, as wide-field calcium imaging does not possess single- cell resolution, the signal in each pixel is an integration of both somatic and neuropil activity. The latter mainly consists of activity from the dense neuropils in layer 1 , including dendrites from local neurons whose somata reside in layers $2 / 3$ and 5 , as well as axons innervating these layer 1 dendrites. Although the majority of wide-field calcium signals reflect local activity (Makino et al., 2017), the contributions of long-range axonal projections are not negligible. Soma-targeting of GCaMP would ensure a cleaner representation of local neural activity in future studies (Y. Chen et al., 2020; Shemesh et al., 2020).

In addition, the raw fluorescence signal of wide-field calcium images is contaminated by hemodynamic changes. The excitation and emission wavelengths of GCaMP reside in the absorption spectrum of oxyhemoglobin and deoxyhemoglobin, so changes in blood oxygenation can contaminate measures of GCaMP fluorescence signals. Currently, there are several methods available to correct hemodynamic contamination. For example, using a secondary wavelength of light allows the estimation of reflectance changes caused by hemodynamics, which can then be used for a regression-based subtraction of hemodynamic signals (Ma et al., 2016a; Wekselblatt et al., 2016; Valley et al., 2020). Low-pass filtering of wide-field signals has also been used to reduce hemodynamic contamination, as hemodynamic artifacts are the strongest in the frequency range corresponding to the heartbeat (Vanni and Murphy, 2014; Xiao et al., 2017). Another analytical correction for hemodynamic signals is to extract hemodynamic components using principal component analysis followed by independent component analysis, and reconstructing the corrected wide-field signals from the remaining components that reflect neural activity (Makino et al., 2017). Alternatively or in addition, repeating the same experiments in animals expressing activity-insensitive GFP can be used as a control to test whether the observed wide-field signals are mainly attributable to neural activity instead of hemodynamic artifacts (Vanni and Murphy, 2014; Wekselblatt et al., 2016).

The temporal resolution of wide-field calcium signals is limited by the relatively slow kinetics of existing calcium indicators. For example, GCaMP6f failed to track synchronous population activity beyond $40 \mathrm{~Hz}$ (Li et al., 2019). Deconvolution of widefield calcium signals can improve the temporal resolution. The heterogeneous spiking activity of many neurons contributing to wide-field calcium signals makes it difficult to generate a general deconvolution algorithm, but attempts are being made to provide the ground truth by simultaneous electrophysiological recordings in the cortex during wide-field calcium imaging (Stern et al., 2020; Peters et al., 2021).

Another issue of consideration arises from parcellation methods used to define cortical regions, as different methods can generate very different results (Barson et al., 2020; Lake et al., 2020). The most common method is to segment the cortex based on an anatomic reference atlas (Wang et al., 2020). The advantage of this approach is the consistency across different studies and research groups, making it convenient to compare results from different studies. However, anatomic reference atlases inevitably ignore individual variations in anatomic structures. Such static atlases also fail to track the dynamic organization of functional cortical modules in different sensory and cognitive processes, which may mask real activity features because of imprecise parcellation (Barson et al., 2020; Saxena et al., 2020). An alternative approach is to define cortical regions based on activity and generate a unique atlas for individual animals. Related methods include grouping pixels using clustering analyses (Barson et al., 2020; Lake et al., 2020) and extracting functional modules using 
non-negative matrix factorization (Saxena et al., 2020) or independent component analysis (Makino et al., 2017). Compared with anatomic atlases, atlases derived from neural activity can more faithfully represent functional organization of the cortex in individual animals. They may also be able to detect neural dynamics localized to regions that do not correspond to standard areas in anatomic atlases. However, functional modules often vary across individual animals and different studies (Makino et al., 2017; Barson et al., 2020; Lake et al., 2020). Different research groups also use different terminologies to refer to regions in their functional atlases. All these factors make it difficult to compare and interpret results across studies. An open platform that allows researchers to register their functional atlases to a common anatomic framework based on coordinates or certain landmarks (e.g., surface blood vessels) would help comparisons across studies.

Finally, as is common in neural recording experiments, caution is warranted in interpreting cortex-wide activity patterns. Functional connectivity and information flows revealed in recent studies using wide-field calcium imaging were extracted by correlational analyses. In these analyses, whether and how cortical regions are connected and influence each other is unclear. Furthermore, cortical regions exhibiting task-related activity may not actually contribute to task performance (Goard et al., 2016; Allen et al., 2017; Pinto et al., 2019; Zatka-Haas et al., 2020). Combining wide-field calcium imaging with activity manipulations (Allen et al., 2017; Makino et al., 2017) and anatomic tracing (Oh et al., 2014) will provide additional insights into causal relationships between cortical regions and their roles in behavior.

\section{Perspectives}

The recent improvements to genetically encoded calcium sensors have resurrected broader interests in using wide-field imaging to investigate large-scale cortical dynamics in behaving animals. As we have discussed above, with wide-field calcium imaging, significant progress has been made to uncover the macroscopic properties of cortical dynamics in various cognitive processes. In the future, we see transformative opportunities for the application of wide-field imaging in the following directions.

\section{Characterizing cell type-specific functions with genetically re- stricted expression of indicators}

The majority of existing studies using wide-field calcium imaging focused on the dynamics of pan-cortical excitatory neurons, but cortical circuits consist of different neuronal types and each carries out distinct functions. For example, cortical excitatory neurons can be further defined by their transcriptomics and anatomic connections, and distinct subpopulations route different information from a specific set of inputs to outputs (Economo et al., 2018; Tasic et al., 2018; Harris et al., 2019). The recent expansion of transgenic mouse lines to target specific subpopulations of excitatory, inhibitory, and modulatory neurons allows genetic targeting of these distinct subpopulations (Madisen et al., 2015; Daigle et al., 2018). By restricting the expression of activity indicators, the monitoring of cell type-specific macroscopic dynamics will dissect the role of different neuronal types and help researchers understand how different components cooperate in cortical circuits at the macroscale. It will also provide valuable datasets for the development of largescale computational models with cell-type resolution.

\section{Macroscopic dynamics of various neurotransmitters}

The nervous system uses a large variety of neurotransmitters and modulators, each of which has unique functions. There has been a recent expansion of toolkits with genetically encoded indicators of various neurotransmitters (Lin and Schnitzer, 2016; Leopold et al., 2019; Dong et al., 2020; Jing et al., 2020; Ravotto et al., 2020; Sabatini and Tian, 2020; F. Sun et al., 2020; Wan et al., 2021; Wu et al., 2020). Wide-field imaging of indicators of specific neurotransmitters/modulators will allow direct tracking of how different molecular signaling is orchestrated at the macroscale. Several pioneering studies have started characterizing cortex-wide patterns of specific neurotransmitters/modulators in spontaneous brain activity (Xie et al., 2016; Lohani et al., 2020). Of particular interest in the future is how different neuromodulatory systems function at the macroscale, because they often project broadly to the cortex and have widespread impacts on behavior and cognition (Avery and Krichmar, 2017).

\section{Expanding toolkits of novel genetically encoded indicators and miniaturized imaging devices}

Genetically encoded indicators with enhanced brightness, sensitivity, stability, and faster kinetics will be fundamental to improving the SNR and temporal resolution of wide-field imaging in future studies. Some recently developed indicators for specific neurotransmitters hold promise for applications in in vivo widefield imaging (Feng et al., 2019; Jing et al., 2020; Lohani et al., 2020; F. Sun et al., 2020). Improvements in voltage indicators could enable future wide-field voltage imaging to capture macroscopic dynamics at millisecond resolution with cell type specificity and longitudinal monitoring (Knöpfel and Song, 2019; Piatkevich et al., 2019; Pal and Tian, 2020). Furthermore, indicators targeting specific subcellular compartments (e.g., soma, axon) will help further determine the relative contributions of different sources in wide-field signals (Broussard et al., 2018; Y. Chen et al., 2020; Shemesh et al., 2020). Meanwhile, broadening the color spectrum of genetically encoded indicators could enable simultaneous imaging of different circuit components and investigations of their interactions (Inoue et al., 2019; Montagni et al., 2019). In addition, virtually all studies with wide-field imaging so far have been performed in head-fixed animals. Miniaturized devices for wide-field imaging in freely-moving animals would uncover large-scale neural dynamics in more naturalistic behavioral contexts (Scott et al., 2018; Adams et al., 2020; Rynes et al., 2021).

\section{Activity manipulations with simultaneous wide-field imaging}

Little is known about how large-scale cortical networks are influenced by individual brain regions and how they might be able to compensate for loss of individual regions. Such interactions between local and global networks can be probed by combining manipulations on certain brain regions or neuronal populations with simultaneous widefield imaging of the cortex. This approach can dissect the functions of individual circuit components and their different contributions in various cognitive processes or at different developmental stages. For example, bilateral M2 inactivation with muscimol combined with simultaneous wide-field calcium imaging uncovered an indispensable role for M2 in orchestrating cortex-wide dynamics acquired with learning (Makino et al., 2017). Manipulating activity in specific areas will also help reveal how large-scale cortical circuits adapt to insults and neurologic disorders. Here a careful comparison between acute (e.g., optogenetics, pharmacogenetics, and pharmacology) and chronic (e.g., lesion) manipulation methods would be critical.

\section{Analysis methods}

The rich dataset obtained by wide-field imaging brings both challenges and unprecedented opportunities to gain insights into 
large-scale cortical dynamics. Sophisticated data-science tools, including dimensionality reduction techniques, will help extract latent and novel patterns from such high dimensional measurements of neural activity and facilitate data-driven discoveries (Williamson et al., 2019). Meanwhile, other analysis tools and computational approaches created for other large-scale recording techniques, such as fMRI and ECoG recordings, can be transferred to wide-field imaging for analyzing network properties (Bressler and Menon, 2010; Rubinov and Sporns, 2010; Pourahmadi and Noorbaloochi, 2016; Cohen et al., 2017). We predict an exponential growth in collaborations between experimental neuroscientists and data scientists to interpret these and other high-dimensional data. For example, creating data-guided circuit models that operate similarly to biological neural networks would provide insights for understanding large-scale cortical networks and in turn guide future experiments (Chaudhuri et al., 2015; Brunton and Beyeler, 2019; Mejias and Wang, 2019).

\section{Conclusion}

Wide-field calcium imaging enables large-scale, unbiased observation of many cortical regions with a sufficient spatiotemporal resolution to capture moment-by-moment features in macroscopic neural dynamics. This technique has started to reveal how cortex-wide dynamics support various cognitive processes, including sensorimotor integration, decision-making, and learning. In addition, combining wide-field calcium imaging with complementary recording modalities provides a novel platform to examine the relationship between local and global neural networks and to characterize the interactions between the cortex and subcortical regions. Although several technical considerations still exist, future applications of wide-field imaging together with rapidly growing data science tools will advance our understanding of how different cell types, neurotransmitters, and brain regions cooperate at the macroscale to give rise to perception and behavior.

\section{References}

Ackman JB, Burbridge TJ, Crair MC (2012) Retinal waves coordinate patterned activity throughout the developing visual system. Nature 490:219225.

Adams J, Boominathan V, Gao S, Rodriguez A, Yan D, Kemere C, Veeraraghavan A, Robinson J (2020) In vivo fluorescence imaging with a flat, lensless microscope. bioRxiv 135236.

Akerboom J, Chen TW, Wardill TJ, Tian L, Marvin JS, Mutlu S, Calderón NC, Esposti F, Borghuis BG, Sun XR, Gordus A, Orger MB, Portugues R, Engert F, Macklin JJ, Filosa A, Aggarwal A, Kerr RA, Takagi R, Kracun S, et al. (2012) Optimization of a GCaMP calcium indicator for neural activity imaging. J Neurosci 32:13819-13840.

Allen WE, Kauvar IV, Chen MZ, Richman EB, Yang SJ, Chan K, Gradinaru V, Deverman BE, Luo L, Deisseroth K (2017) Global representations of goal-directed behavior in distinct cell types of mouse neocortex. Neuron 94:891-907.e6.

Avery MC, Krichmar JL (2017) Neuromodulatory systems and their interactions: a review of models, theories, and experiments. Front Neural Circuits 11:108.

Barson D, Hamodi AS, Shen X, Lur G, Constable RT, Cardin JA, Crair MC, Higley MJ (2020) Simultaneous mesoscopic and two-photon imaging of neuronal activity in cortical circuits. Nat Methods 17:107-113.

Bauer AQ, Kraft AW, Wright PW, Snyder AZ, Lee JM, Culver JP (2014) Optical imaging of disrupted functional connectivity following ischemic stroke in mice. Neuroimage 99:388-401.

Berwick J, Johnston D, Jones M, Martindale J, Redgrave P, McLoughlin N, Schiessl I, Mayhew JE (2005) Neurovascular coupling investigated with two-dimensional optical imaging spectroscopy in rat whisker barrel cortex. Eur J Neurosci 22:1655-1666.
Blasdel GG, Salama G (1986) Voltage-sensitive dyes reveal a modular organization in monkey striate cortex. Nature 321:579-585.

Bonhoeffer T, Grinvald A (1991) Iso-orientation domains in cat visual cortex are arranged in pinwheel-like patterns. Nature 353:429-431.

Bressler SL, Menon V (2010) Large-scale brain networks in cognition: emerging methods and principles. Trends Cogn Sci 14:277-290.

Broussard GJ, Liang Y, Fridman M, Unger EK, Meng G, Xiao X, Ji N, Petreanu L, Tian L (2018) In vivo measurement of afferent activity with axon-specific calcium imaging. Nat Neurosci 21:1272-1280.

Brunton BW, Beyeler M (2019) Data-driven models in human neuroscience and neuroengineering. Curr Opin Neurobiol 58:21-29.

Burbridge TJ, Xu HP, Ackman JB, Ge X, Zhang Y, Ye MJ, Zhou ZJ, Xu J, Contractor A, Crair MC (2014) Visual circuit development requires patterned activity mediated by retinal acetylcholine receptors. Neuron 84:1049-1064.

Cardin JA, Crair MC, Higley MJ (2020) Mesoscopic imaging: shining a wide light on large-scale neural dynamics. Neuron 108:33-43.

Chaudhuri R, Knoblauch K, Gariel MA, Kennedy H, Wang XJ (2015) A large-scale circuit mechanism for hierarchical dynamical processing in the primate cortex. Neuron 88:419-431.

Chen TW, Wardill TJ, Sun Y, Pulver SR, Renninger SL, Baohan A, Schreiter ER, Kerr RA, Orger MB, Jayaraman V, Looger LL, Svoboda K, Kim DS (2013) Ultrasensitive fluorescent proteins for imaging neuronal activity. Nature 499:295-300.

Chen TW, Li N, Daie K, Svoboda K (2017) A map of anticipatory activity in mouse motor cortex. Neuron 94:866-879.e4.

Chen Y, Jang H, Spratt PW, Kosar S, Taylor DE, Essner RA, Bai L, Leib DE, Kuo TW, Lin YC, Patel M, Subkhangulova A, Kato S, Feinberg EH, Bender KJ, Knight ZA, Garrison JL (2020) Soma-targeted imaging of neural circuits by ribosome tethering. Neuron 107:454-469.e6.

Clancy KB, Orsolic I, Mrsic-Flogel TD (2019) Locomotion-dependent remapping of distributed cortical networks. Nat Neurosci 22:778-786.

Cohen JD, Daw N, Engelhardt B, Hasson U, Li K, Niv Y, Norman KA, Pillow J, Ramadge PJ, Turk-Browne NB, Willke TL (2017) Computational approaches to fMRI analysis. Nat Neurosci 20:304-313.

Costa RM, Dana C, Nicolelis MA (2004) Differential corticostriatal plasticity during fast and slow motor skill learning in mice. Curr Biol 14:11241134.

Daigle TL, Madisen L, Hage TA, Valley MT, Knoblich U, Larsen RS, Takeno MM, Huang L, Gu H, Larsen R, Mills M, Bosma-Moody A, Siverts LA, Walker M, Graybuck LT, Yao Z, Fong O, Nguyen TN, Garren E, Lenz $\mathrm{GH}$, et al. (2018) A suite of transgenic driver and reporter mouse lines with enhanced brain-cell-type targeting and functionality. Cell 174:465480.e22.

Dana H, Sun Y, Mohar B, Hulse BK, Kerlin AM, Hasseman JP, Tsegaye G, Tsang A, Wong A, Patel R, Macklin JJ, Chen Y, Konnerth A, Jayaraman V, Looger LL, Schreiter ER, Svoboda K, Kim DS (2019) High-performance calcium sensors for imaging activity in neuronal populations and microcompartments. Nat Methods 16:649-657.

Dong A, He K, Dudok B, Farrell JS, Guan W, Liput DJ, Puhl HL, Cai R, Duan J, Albarran E, Ding J, Lovinger DM, Li B, Soltesz I, Li Y (2020) A fluorescent sensor for spatiotemporally resolved endocannabinoid dynamics in vitro and in vivo. bioRxiv 329169.

Economo MN, Viswanathan S, Tasic B, Bas E, Winnubst J, Menon V, Graybuck LT, Nguyen TN, Smith KA, Yao Z, Wang L, Gerfen CR, Chandrashekar J, Zeng H, Looger LL, Svoboda K (2018) Distinct descending motor cortex pathways and their roles in movement. Nature 563:79-84.

Feng J, Zhang C, Lischinsky JE, Jing M, Zhou J, Wang H, Zhang Y, Dong A, Wu Z, Wu H, Chen W, Zhang P, Zou J, Hires SA, Zhu JJ, Cui G, Lin D, Du J, Li Y (2019) A genetically encoded fluorescent sensor for rapid and specific in vivo detection of norepinephrine. Neuron 102:745-761.e8.

Ferezou I, Haiss F, Gentet LJ, Aronoff R, Weber B, Petersen CC (2007) Spatiotemporal dynamics of cortical sensorimotor integration in behaving mice. Neuron 56:907-923.

Frostig RD, Lieke EE, Ts'o DY, Grinvald A (1990) Cortical functional architecture and local coupling between neuronal activity and the microcirculation revealed by in vivo high-resolution optical imaging of intrinsic signals. Proc Natl Acad Sci USA 87:6082-6086.

Garrett ME, Nauhaus I, Marshel JH, Callaway EM, Garrett ME, Marshel JH, Nauhaus I, Garrett ME (2014) Topography and areal organization of mouse visual cortex. J Neurosci 34:12587-12600. 
Gilad A, Gallero-Salas Y, Groos D, Helmchen F (2018) Behavioral strategy determines frontal or posterior location of short-term memory in neocortex. Neuron 99:814-828.e7.

Gilad A, Helmchen F (2020) Spatiotemporal refinement of signal flow through association cortex during learning. Nat Commun 11:1744.

Goard MJ, Pho GN, Woodson J, Sur M (2016) Distinct roles of visual, parietal, and frontal motor cortices in memory-guided sensorimotor decisions. Elife 5:e13764.

Grewe BF, Gründemann J, Kitch LJ, Lecoq JA, Parker JG, Marshall JD, Larkin MC, Jercog PE, Grenier F, Li JZ, Lüthi A, Schnitzer MJ (2017) Neural ensemble dynamics underlying a long-term associative memory. Nature 543:670-675.

Gribizis A, Ge X, Daigle TL, Ackman JB, Zeng H, Lee D, Crair MC (2019) Visual cortex gains independence from peripheral drive before eye opening. Neuron 104:711-723.e3.

Grinvald A, Hildesheim R (2004) VSDI: a new era in functional imaging of cortical dynamics. Nat Rev Neurosci 5:874-885

Grinvald A, Lieke E, Frostig RD, Gilbert CD, Wiesel TN (1986) Functional architecture of cortex revealed by optical imaging of intrinsic signals. Nature 324:361-363.

Guo ZV, Li N, Huber D, Ophir E, Gutnisky D, Ting JT, Feng G, Svoboda K (2014) Flow of cortical activity underlying a tactile decision in mice. Neuron 81:179-194.

Harris JA, Mihalas S, Hirokawa KE, Whitesell JD, Choi H, Bernard A, Bohn P, Caldejon S, Casal L, Cho A, Feiner A, Feng D, Gaudreault N, Gerfen CR, Graddis N, Groblewski PA, Henry AM, Ho A, Howard R, Knox JE, et al. (2019) Hierarchical organization of cortical and thalamic connectivity. Nature 575:195-202.

Hattori R, Danskin B, Babic Z, Mlynaryk N, Komiyama T (2019) Area-specificity and plasticity of history-dependent value coding during learning. Cell 177:1858-1872.e15.

Inoue M, Takeuchi A, Manita S, Horigane SI, Sakamoto M, Kawakami R, Yamaguchi K, Otomo K, Yokoyama H, Kim R, Yokoyama T, TakemotoKimura S, Abe M, Okamura M, Kondo Y, Quirin S, Ramakrishnan C, Imamura T, Sakimura K, Nemoto T, et al. (2019) Rational engineering of XCaMPs, a multicolor GECI suite for in vivo imaging of complex brain circuit dynamics. Cell 177:1346-1360.e24.

Ji N, Freeman J, Smith SL (2016) Technologies for imaging neural activity in large volumes. Nat Neurosci 19:1154-1164.

Jing M, Li Y, Zeng J, Huang P, Skirzewski M, Kljakic O, Peng W, Qian T, Tan K, Zou J, Trinh S, Wu R, Zhang S, Pan S, Hires SA, Xu M, Li H, Saksida LM, Prado VF, Bussey TJ, et al. (2020) An optimized acetylcholine sensor for monitoring in vivo cholinergic activity. Nat Methods 17:1139-1146.

Jung WB, Shim HJ, Kim SG (2019) Mouse BOLD fMRI at ultrahigh field detects somatosensory networks including thalamic nuclei. Neuroimage 195:203-214.

Karimi Abadchi J, Nazari-Ahangarkolaee M, Gattas S, Bermudez-Contreras E, Luczak A, McNaughton BL, Mohajerani MH (2020) Spatiotemporal patterns of neocortical activity around hippocampal sharp-wave ripples. Elife 9:1-26.

Knöpfel T, Song C (2019) Optical voltage imaging in neurons: moving from technology development to practical tool. Nat Rev Neurosci 20:719-727.

Kura S, Xie H, Fu B, Ayata C, Boas DA, Sakadzõić S (2018) Intrinsic optical signal imaging of the blood volume changes is sufficient for mapping the resting state functional connectivity in the rodent cortex. J Neural Eng 15:035003

Kyriakatos A, Sadashivaiah V, Zhang Y, Motta A, Auffret M, Petersen CC, Petersen C (2017) Voltage-sensitive dye imaging of mouse neocortex during a whisker detection task. Neurophotonics 4:031204.

Lake EM, Ge X, Shen X, Herman P, Hyder F, Cardin JA, Higley MJ, Scheinost D, Papademetris X, Crair MC, Constable RT (2020) Simultaneous cortex-wide fluorescence $\mathrm{Ca}^{2+}$ imaging and whole-brain fMRI. Nat Methods 17:1262-1271.

Leopold AV, Shcherbakova DM, Verkhusha VV (2019) Fluorescent biosensors for neurotransmission and neuromodulation: engineering and applications. Front Cell Neurosci 13:474.

Li P, Geng X, Jiang H, Caccavano A, Vicini S, Wu JY (2019) Measuring sharp waves and oscillatory population activity with the genetically encoded calcium indicator GCaMP6f. Front Cell Neurosci 13:274.

Lin MZ, Schnitzer MJ (2016) Genetically encoded indicators of neuronal activity. Nat Neurosci 19:1142-1153.
Liu X, Ren C, Lu Y, Liu Y, Kim J, Leutgeb S, Komiyama T, Kuzum D (2021) Multimodal neural recordings with Neuro-FITM uncover diverse patterns of cortical-hippocampal interactions. Nat Neurosci. Advance online publication. Retrieved April 19, 20201. doi: 10.1038/s41593-021-00841-5.

Lohani S, Moberly AH, Benisty H, Landa B, Jing M, Li Y, Higley MJ, Cardin JA (2020) Dual color mesoscopic imaging reveals spatiotemporally heterogeneous coordination of cholinergic and neocortical activity. bioRxiv 418632

Lu R, Liang Y, Meng G, Zhou P, Svoboda K, Paninski L, Ji N (2020) Rapid mesoscale volumetric imaging of neural activity with synaptic resolution. Nat Methods 17:291-294.

Ma Y, Shaik MA, Kim SH, Kozberg MG, Thibodeaux DN, Zhao HT, Yu H, Hillman EMC (2016a) High-speed, wide-field optical mapping (WFOM) of neural activity and brain haemodynamics: considerations and novel approaches. Philos Trans R Soc Lond B Biol Sci 371:20150360.

Ma Y, Shaik MA, Kozberg MG, Kim SH, Portes JP, Timerman D, Hillman EMC (2016b) Resting-state hemodynamics are spatiotemporally coupled to synchronized and symmetric neural activity in excitatory neurons. Proc Natl Acad Sci USA 113:E8463-E8471.

Madisen L, Garner AR, Shimaoka D, Chuong AS, Klapoetke NC, Li L, van der Bourg A, Niino Y, Egolf L, Monetti C, Gu H, Mills M, Cheng A, Tasic B, Nguyen TN, Sunkin SM, Benucci A, Nagy A, Miyawaki A, Helmchen F, et al. (2015) Transgenic mice for intersectional targeting of neural sensors and effectors with high specificity and performance. Neuron 85:942-958.

Makino H, Komiyama T (2015) Learning enhances the relative impact of top-down processing in the visual cortex. Nat Neurosci 18:1116-1122.

Makino H, Ren C, Liu H, Kim AN, Kondapaneni N, Liu X, Kuzum D, Komiyama T (2017) Transformation of cortex-wide emergent properties during motor learning. Neuron 94:880-890.e8.

Mateo C, Knutsen PM, Tsai PS, Shih AY, Kleinfeld D (2017) Entrainment of arteriole vasomotor fluctuations by neural activity is a basis of blood-oxygenation-level-dependent "resting-state" connectivity. Neuron 96:936948.e3.

Matyas F, Sreenivasan V, Marbach F, Wacongne C, Barsy B, Mateo C, Aronoff R, Petersen CC (2010) Motor control by sensory cortex. Science 330:1240-1244.

Mejias J, Wang XJ (2019) Mechanisms of distributed working memory in a large-scale model of macaque neocortex. bioRxiv $760231 .$.

Mohajerani MH, McVea DA, Fingas M, Murphy TH (2010) Mirrored bilateral slow-wave cortical activity within local circuits revealed by fast bihemispheric voltage-sensitive dye imaging in anesthetized and awake mice. J Neurosci 30:3745-3751.

Montagni E, Resta F, Conti E, Scaglione A, Pasquini M, Micera S, Mascaro AL, Pavone FS (2019) Wide-field imaging of cortical neuronal activity with red-shifted functional indicators during motor task execution. J Phys D 52:074001

Musall S, Kaufman MT, Juavinett AL, Gluf S, Churchland AK (2019) Singletrial neural dynamics are dominated by richly varied movements. Nat Neurosci 22:1677-1686.

Niethard N, Hasegawa M, Itokazu T, Oyanedel CN, Born J, Sato TR (2016) Sleep-stage-specific regulation of cortical excitation and inhibition. Curr Biol 26:2739-2749.

Nöbauer T, Skocek O, Pernía-Andrade AJ, Weilguny L, Martínez Traub F, Molodtsov MI, Vaziri A (2017) Video rate volumetric $\mathrm{Ca}^{2+}$ imaging across cortex using seeded iterative demixing (SID) microscopy. Nat Methods 14:811-818.

Oh SW, Harris JA, Ng L, Winslow B, Cain N, Mihalas S, Wang Q, Lau C, Kuan L, Henry AM, Mortrud MT, Ouellette B, Nguyen TN, Sorensen SA, Slaughterbeck CR, Wakeman W, Li Y, Feng D, Ho A, Nicholas E, et al. (2014) A mesoscale connectome of the mouse brain. Nature 508:207214.

Orbach HS, Cohen LB, Grinvald A (1985) Optical mapping of electrical activity in rat somatosensory and visual cortex. J Neurosci 5:1886-1895.

Ota K (2020) Fast scanning high optical invariant two-photon microscopy for monitoring a large neural network activity with cellular resolution. bioRxiv 201699.

Pal A, Tian L (2020) Imaging voltage and brain chemistry with genetically encoded sensors and modulators. Curr Opin Chem Biol 57:166-176.

Peters AJ, Chen SX, Komiyama T (2014) Emergence of reproducible spatiotemporal activity during motor learning. Nature 510:263-267. 
Peters AJ, Fabre JM, Steinmetz NA, Harris KD, Carandini M (2021) Striatal activity topographically reflects cortical activity. Nature 591:420-425.

Piatkevich KD, Bensussen S, Tseng HA, Shroff SN, Lopez-Huerta VG, Park D, Jung EE, Shemesh OA, Straub C, Gritton HJ, Romano MF, Costa E, Sabatini BL, Fu Z, Boyden ES, Han X (2019) Population imaging of neural activity in awake behaving mice. Nature 574:413-417.

Pinto L, Rajan K, DePasquale B, Thiberge SY, Tank DW, Brody CD (2019) Task-dependent changes in the large-scale dynamics and necessity of cortical regions. Neuron 104:810-824.e9.

Pourahmadi M, Noorbaloochi S (2016) Multivariate time series analysis of neuroscience data: some challenges and opportunities. Curr Opin Neurobiol 37:12-15.

Prechtl JC, Cohen LB, Pesaran B, Mitra PP, Kleinfeld D (1997) Visual stimuli induce waves of electrical activity in turtle cortex. Proc Natl Acad Sci USA 94:7621-7626.

Ravotto L, Duffet L, Zhou X, Weber B, Patriarchi T (2020) A bright and colorful future for G-protein coupled receptor sensors. Front Cell Neurosci 14:1-9.

Rubinov M, Sporns O (2010) Complex network measures of brain connectivity: uses and interpretations. Neuroimage 52:1059-1069.

Rynes M, Surinach D, Laroque M, Linn S, Dominguez J, Ghanbari L, Kodandaramaiah S (2021) Miniaturized device for whole cortex mesoscale imaging in freely behaving mice. Nat Methods. Advance online publication. Retrieved April 5, 2021. doi: 10.1038/s41592-021-01104-8.

Sabatini BL, Tian L (2020) Imaging neurotransmitter and neuromodulator dynamics in vivo with genetically encoded indicators. Neuron 108:17-32.

Salkoff DB, Zagha E, McCarthy E, McCormick DA (2020) Movement and performance explain widespread cortical activity in a visual detection task. Cereb Cortex 30:421-437.

Saxena S, Kinsella I, Musall S, Kim SH, Meszaros J, Thibodeaux DN, Kim C, Cunningham J, Hillman EM, Churchland A, Paninski L (2020) Localized semi-nonnegative matrix factorization (LocaNMF) of widefield calcium imaging data. PLoS Comput Biol 16:e1007791.

Schlegel F, Sych Y, Schroeter A, Stobart J, Weber B, Helmchen F, Rudin M (2018) Fiber-optic implant for simultaneous fluorescence-based calcium recordings and BOLD fMRI in mice. Nat Protoc 13:840-855.

Schwalm M, Schmid F, Wachsmuth L, Backhaus H, Kronfeld A, Jury FA, Prouvot PH, Fois C, Albers F, Van Alst T, Faber C, Stroh A (2017) Cortex-wide BOLD fMRI activity reflects locally-recorded slow oscillation-associated calcium waves. Elife 6:e27602.

Scott BB, Thiberge SY, Guo C, Tervo DG, Brody CD, Karpova AY, Tank DW (2018) Imaging cortical dynamics in GCaMP transgenic rats with a head-mounted widefield macroscope. Neuron 100:1045-1058.e5.

Shemesh OA, Linghu C, Piatkevich KD, Goodwin D, Celiker OT, Gritton HJ, Romano MF, Gao R, Yu CC, Tseng HA, Bensussen S, Narayan S, Yang CT, Freifeld L, Siciliano CA, Gupta I, Wang J, Pak N, Yoon YG, Ullmann JF, et al. (2020) Precision calcium imaging of dense neural populations via a cell-body-targeted calcium indicator. Neuron 107:470-486.e11.

Shimaoka D, Steinmetz NA, Harris KD, Carandini M (2019) The impact of bilateral ongoing activity on evoked responses in mouse cortex. Elife 8: e43533.

Sofroniew NJ, Flickinger D, King J, Svoboda K (2016) A large field of view two-photon mesoscope with subcellular resolution for in vivo imaging. Elife 5:e14472.

Song A, Charles AS, Koay SA, Gauthier JL, Thiberge SY, Pillow JW, Tank DW (2017) Volumetric two-photon imaging of neurons using stereoscopy (vTwINS). Nat Methods 14:420-426.

Sreenivasan V, Kyriakatos A, Mateo C, Jaeger D, Petersen CC (2017) Parallel pathways from whisker and visual sensory cortices to distinct frontal regions of mouse neocortex. Neurophotonics 4:031203.

Steinmetz NA, Zatka-Haas P, Carandini M, Harris KD (2019) Distributed coding of choice, action and engagement across the mouse brain. Nature 576:266-273.

Stern M, Shea-Brown E, Witten D (2020) Inferring the spiking rate of a population of neurons from wide-field calcium imaging. bioRxiv 930040.

Stirman JN, Smith IT, Kudenov MW, Smith SL (2016) Wide field-of-view, multi-region two-photon imaging of neuronal activity. Nat Biotechnol 34:857-870.

Stringer C, Pachitariu M, Steinmetz N, Reddy CB, Carandini M, Harris KD (2019) Spontaneous behaviors drive multidimensional, brainwide activity. Science 364:255
Sun F, Zhou J, Dai B, Qian T, Zeng J, Li X, Zhuo Y, Zhang Y, Wang Y, Qian C, Tan K, Feng J, Dong H, Lin D, Cui G, Li Y (2020) Next-generation GRAB sensors for monitoring dopaminergic activity in vivo. Nat Methods 17:1156-1166.

Sun XR, Badura A, Pacheco DA, Lynch LA, Schneider ER, Taylor MP, Hogue IB, Enquist LW, Murthy M, Wang SS (2013) Fast GCaMPs for improved tracking of neuronal activity. Nat Commun 4:2170.

Tasic B, Yao Z, Graybuck LT, Smith KA, Nguyen TN, Bertagnolli D, Goldy J, Garren E, Economo MN, Viswanathan S, Penn O, Bakken T, Menon V, Miller J, Fong O, Hirokawa KE, Lathia K, Rimorin C, Tieu M, Larsen R, et al. (2018) Shared and distinct transcriptomic cell types across neocortical areas. Nature 563:72-78.

Tian L, Hires SA, Mao T, Huber D, Chiappe ME, Chalasani SH, Petreanu L, Akerboom J, McKinney SA, Schreiter ER, Bargmann CI, Jayaraman V, Svoboda K, Looger LL (2009) Imaging neural activity in worms, flies and mice with improved GCaMP calcium indicators. Nat Methods 6:875-881.

Valley MT, Moore MG, Zhuang J, Mesa N, Castelli D, Sullivan D, Reimers M, Waters J (2020) Separation of hemodynamic signals from GCaMP fluorescence measured with wide-field imaging. J Neurophysiol 123:356-366.

Vanni MP, Murphy TH (2014) Mesoscale transcranial spontaneous activity mapping in GCaMP3 transgenic mice reveals extensive reciprocal connections between areas of somatomotor cortex. J Neurosci 34:1593115946.

Wan J, Peng W, Li X, Qian T, Song K, Zeng J, Deng F, Hao S, Feng J, Zhang P, Zhang Y, Zou J, Pan S, Zhu JJ, Jing M, Xu M, Li Y (2021) A genetically encoded GRAB sensor for measuring serotonin dynamics in vivo. Nat Neurosci. Advance online publication. Retrieved April 5, 2021. doi: 10.1038/s41593-021-00823-7.

Wang Q, Ding SL, Li Y, Royall J, Feng D, Lesnar P, Graddis N, Naeemi M, Facer B, Ho A, Dolbeare T, Blanchard B, Dee N, Wakeman W, Hirokawa KE, Szafer A, Sunkin SM, Oh SW, Bernard A, Phillips JW, et al. (2020) The Allen mouse brain common coordinate framework: a 3D reference atlas. Cell 181:936-953.e20.

Weisenburger S, Tejera F, Demas J, Chen B, Manley J, Sparks FT, Martínez Traub F, Daigle T, Zeng H, Losonczy A, Vaziri A (2019) Volumetric $\mathrm{Ca}^{2+}$ imaging in the mouse brain using hybrid multiplexed sculpted light microscopy. Cell 177:1050-1066.e14.

Wekselblatt JB, Flister ED, Piscopo DM, Niell CM (2016) Large-scale imaging of cortical dynamics during sensory perception and behavior. J Neurophysiol 115:2852-2866

Williamson RC, Doiron B, Smith MA, Yu BM (2019) Bridging large-scale neuronal recordings and large-scale network models using dimensionality reduction. Curr Opin Neurobiol 55:40-47.

Wu Z, Cui Y, Wang H, Song K, Yuan Z, Dong A, Wu H, Wan Y, Pan S, Peng W, Jing M, Xu M, Luo M, Li Y (2020) A GRAB sensor reveals activity-dependent non-vesicular somatodendritic adenosine release. bioRxiv 075564.

Xiao D, Vanni MP, Mitelut CC, Chan AW, LeDue JM, Xie Y, Chen AC, Swindale NV, Murphy TH (2017) Mapping cortical mesoscopic networks of single spiking cortical or sub-cortical neurons. Elife 6:e19976.

Xie Y, Chan AW, McGirr A, Xue S, Xiao D, Zeng H, Murphy TH (2016) Resolution of high-frequency mesoscale intracortical maps using the genetically encoded glutamate sensor iGluSnFR. J Neurosci 36:1261-1272.

Yang W, Carrillo-Reid L, Bando Y, Peterka DS, Yuste R (2018) Simultaneous two-photon imaging and two-photon optogenetics of cortical circuits in three dimensions. Elife 7:e32671.

Yang Y, Liu N, He Y, Liu Y, Ge L, Zou L, Song S, Xiong W, Liu X (2018) Improved calcium sensor GCaMP-X overcomes the calcium channel perturbations induced by the calmodulin in GCaMP. Nat Commun 9:1504.

Yizhar O, Fenno LE, Davidson TJ, Mogri M, Deisseroth K (2011) Optogenetics in neural systems. Neuron 71:9-34.

Yu CH, Stirman JN, Yu Y, Hira R, Smith SL (2020) Diesel2p mesoscope with dual independent scan engines for flexible capture of dynamics in distributed neural circuitry. bioRxiv 305508

Zatka-Haas P, Steinmetz NA, Carandini M, Harris KD (2020) A perceptual decision requires sensory but not action coding in mouse cortex. bioRxiv 501627

Zhuang J, Ng L, Williams D, Valley M, Li Y, Garrett M, Waters J (2017) An extended retinotopic map of mouse cortex. Elife 6:e18372. 